\title{
Anti-autophagic and anti-apoptotic effects of memantine in a SH-SY5Y cell model of Alzheimer's disease via mammalian target of rapamycin-dependent and -independent pathways
}

\author{
GUIJUN SONG, YU LI, LULU LIN and YUNPENG CAO
}

Department of Neurology, The First Affiliated Hospital of China Medical University, Shenyang, Liaoning 110001, P.R. China

Received October 17, 2014; Accepted July 17, 2015

DOI: $10.3892 / \mathrm{mmr} .2015 .4382$

\begin{abstract}
Memantine non-competitively blocks the $N$-methyl-D-aspartate receptor in order to inhibit beta-amyloid (A $\beta$ ) secretion, and has been used to treat moderate-to-severe Alzheimer's disease (AD). However, the mechanisms underlying the role of memantine in the autophagy and apoptosis of neuronal cells in $\mathrm{AD}$, as well as the association between neuronal autophagy and apoptosis have yet to be elucidated. The present study aimed to establish an AD cell model overexpressing the 695-amino-acid Swedish mutant of A $\beta$ precursor protein (APP695swe) in order to observe the effects of memantine on the cell viability, autophagy and apoptosis of SH-SY5Y cells in the AD model, and to investigate the associated underlying mechanisms. A pcDNA3.1-APP695 plasmid was transfected into the SH-SY5Y cells. Reverse transcription-quantitative polymerase chain reaction and western blot analyses demonstrated that the AD cell model was successfully established. MTT assays demonstrated that memantine was able to upregulate neuronal cell survival, and acridine orange staining and flow cytometry demonstrated that memantine $(5 \mu \mathrm{M})$ was able to inhibit neuronal autophagy and apoptosis. Following neuronal autophagy induction by rapamycin, cell apoptosis rates increased significantly. Further experiments revealed that memantine was able to upregulate the expression of signaling molecules phosphorylated (p)-phosphoinositide 3-kinase, p-Akt and p-mammalian target of rapamycin (mTOR), and also inhibited the phosphorylation of the B-cell lymphoma 2/Beclin-1 complex via mitogen-activated protein kinase 8 . In conclusion, the results of the present study demonstrated that in the AD cell model, autophagy was able to promote apoptosis. Memantine exerted anti-autophagic and anti-apoptotic functions, and mTOR-dependent as well as
\end{abstract}

Correspondence to: Professor Yunpeng Cao, Department of Neurology, The First Affiliated Hospital of China Medical University, 155 North Nanjing Street, Shenyang, Liaoning 110001, P.R. China

E-mail: yunpengcao@outlook.com

Key words: memantine, APP695, autophagy, apoptosis, mammalian target of rapamycin -independent autophagic signaling pathways were involved in this process. Therefore, these results of the present study strongly supported the use of memantine as a potential therapeutic strategy for AD treatment.

\section{Introduction}

Alzheimer's disease (AD) is a progressive neurodegenerative disorder characterized by cognitive and memory deterioration, changes in personality, behavioral disturbances and an impaired ability to perform daily living activities (1). The number of patients with AD is increasing every year. Therefore, an effective therapeutic drug is still an urgent requirement for the treatment of AD (2). Extracellular senile plaque (SP) deposition is one of the pathological markers in the brains of patients with AD. SP originates from the abnormal extracellular beta-amyloid (A $\beta)$ aggregation caused by gene mutations in the $\mathrm{A} \beta$ precursor protein (APP). A previous study demonstrated that $\mathrm{A} \beta$ aggregation activated the $N$-methyl-D-aspartate (NMDA) receptor $(3,4)$, induced neurotoxicity and caused neuronal apoptosis and degenerative death (5). Therefore, developing drugs that inhibit neuronal death is important for delaying AD progression.

Memantine is a non-competitive NMDA receptor antagonist, which has been approved for the treatment of moderate-to-severe AD. Numerous clinical trials reported that memantine was able to improve attention, cognitive ability and functional communication in patients with AD (6-11). In vitro studies demonstrated that memantine reduced the secretion of APP and A $\beta$ in APP/PS1 transgenic mice, cultured cortical cells and SK-N-SH human neuroblastoma cells $(12,13)$. Further studies also demonstrated that memantine promotes the proliferation of neural progenitor cells and the production of mature granular neurons in the adult hippocampus (14-16). However, the effects of memantine on neuronal autophagy and apoptosis remain to be elucidated. In addition, it remains controversial whether autophagy promotes $A \beta$ production (17-20) or reduces A $\beta$ levels (21-24).

The present study established an AD cell model overexpressing APP with the 695-amino-acid Swedish mutation (APP695) to determine the effects of memantine on cell autophagy and apoptosis, and to explore the association between autophagy and apoptosis, as well as the underlying mechanisms and signaling pathways involved. 


\section{Materials and methods}

Cell culture and establishment of an AD cell model. The SH-SY5Y human neuroblastoma cell line was purchased from the Shanghai Institute for Biological Sciences of the Chinese Academy of Sciences (Shanghai, China). The cells were cultured in Dulbecco's modified Eagle's medium (Gibco-BRL, Invitrogen Life Technologies, Carlsbad, CA, USA) supplemented with $10 \%$ fetal bovine serum (GE Healthcare Life Sciences, Little Chalfont, UK) and streptomycin/penicillin (100 U/ml) (China National Medicines Corporation, Ltd., Beijing, China) at $37^{\circ} \mathrm{C}$ in an atmosphere containing $5 \% \mathrm{CO}_{2}$. The AD cell model was established a previously described $(25,26)$. When the SH-SY5Y cells reached $70 \%$ confluence, the cells were transfected with overexpression plasmid pcDNA3.1-APP695, containing a Swedish mutant APP695 gene (Wanleibio, Shenyang, China) using the Lipofectamine ${ }^{\circledR} 2000$ reagent (Invitrogen Life Technologies) according to the manufacturer's instructions. The cells were separated into the following groups: A non-transfected control group (Control), a transfection reagent-treated group (Mock), an empty plasmid transfection group (pcDNA3.1) and a pcDNA31-APP695-transfected group (APP695). The cells were collected after 24 or $48 \mathrm{~h}$ of transfection for subsequent experiments.

Drug treatment. Memantine hydrochloride (Sigma-Aldrich, St. Louis, MO, USA) was dissolved in double distilled (dd) $\mathrm{H}_{2} \mathrm{O}$. Rapamycin (RAPA; Melone Pharmaceutical Co., Ltd., Dalian, China) was dissolved in dimethyl sulfoxide (DMSO; Sigma-Aldrich). After 24 or $48 \mathrm{~h}$ of transfection, the cells were treated with various concentrations of memantine $(1,5$ and $10 \mu \mathrm{M})$ to select an optimal concentration for subsequent experiments. For enhanced autophagy experiments, the cells were pre-treated with $0.2 \mu \mathrm{M}$ RAPA for $24 \mathrm{~h}$ prior to treatment with $5 \mu \mathrm{M}$ memantine for $24 \mathrm{~h}$.

Reverse transcription-quantitative polymerase chain reaction $(R T-q P C R)$. At $24 \mathrm{~h}$ post-transfection, the cells were harvested and total RNA from each sample was extracted using a RNA simple Total RNA kit (cat no. DP419; Tiangen Biotech Co., Ltd., Beijing, China). The RNA was then re-suspended in $40 \mu \mathrm{l}$ RNase-free $\mathrm{dd}_{2} \mathrm{O}$ and was reverse transcribed into cDNA using the PrimeScript ${ }^{\mathrm{TM}}$ RT reagent kit (cat no. RR037A; Takara Bio Inc., Dalian, China). Fluorescence quantitative analysis was performed with a $S Y B R^{\circledR}$ GREEN MasterMix (Tiangen Biotech Co., Ltd.) using an Exicycler ${ }^{\mathrm{TM}} 96$ quantitative fluorescence analyzer (Bioneer Corporation, Daejeon, Korea). The thermocycling conditions were set as follows: $95^{\circ} \mathrm{C}$ for $10 \mathrm{~min}$; 40 cycles of $95^{\circ} \mathrm{C}$ for $10 \mathrm{sec}, 60^{\circ} \mathrm{C}$ for $20 \mathrm{sec}$ and $72^{\circ} \mathrm{C}$ for $30 \mathrm{sec}$; and final elongation at $4^{\circ} \mathrm{C}$ for $5 \mathrm{~min}$. $\beta$-actin was included in each reaction as an internal control, and the relative gene expression levels were calculated using the $2^{-\Delta \Delta \mathrm{Ct}}$ method (27). The primer sequences for the RT-qPCR were as follows: APP695 sense, 5'-GGACGATGAGGATGGTGATGAG-3' and anti-sense, 5'-GGTACTGGCTGCTGTTGTAGGA-3'; $\beta$-actin sense, 5'-CTTAGTTGCGTTACACCCTTTCTTG-3' and anti-sense, 5'-CTGTCACCTTCACCGTTCCAGTTT-3'. PCR primers were synthesized by Sangon Biotech (Shanghai, China).
Western blotanalysis. Following $48 \mathrm{~h}$ of transfection, total protein from each group was extracted using NP-40 lysate (Beyotime Institute of Biotechnology, Haimen, China) with $1 \%$ phenylmethanesulfonyl fluoride (Beyotime Institute of Biotechnology). The protein concentration of each sample was determined using a Bicinchoninic Acid Protein Assay kit (cat no. P0012S; Beyotime Institute of Biotechnology). A total of $40 \mu \mathrm{g}$ protein from each group was separated by SDS-PAGE (5\% for the spacer gel and $8 \%$ for the separating gel) and transferred onto polyvinylidene fluoride membranes (EMD Millipore, Billerica, MA, USA). The membranes were blocked with 5\% non-fat milk overnight and incubated at $4{ }^{\circ} \mathrm{C}$ overnight with the following primary antibodies: Rabbit polyclonal anti-APP695 (1:500; cat no. BA0581; Wuhan Boster Biological Technology, Ltd., Wuhan, China), rabbit polyclonal anti-caspase-3 (1:1,000; cat no. bs-0081R; BIOSS, Beijing, China), rabbit polyclonal anti-light chain 3 (LC3; 1:1,000; cat no. 4108; Cell Signaling Technology, Inc., Danvers, MA, USA), rabbit polyclonal anti-autophagy protein 5 (ATG5; 1:200; cat no. BA3525-2; Wuhan Boster Biological Technology, Ltd.), rabbit polyclonal immunoglobulin (Ig)G to phosphorylated (p)-Akt (1:200; cat no. sc-135650; Santa Cruz Biotechnology Inc., Dallas, TX, USA), rabbit polyclonal IgG to B-cell lymphoma 2 (Bcl-2; 1:200; cat no. sc-492; Santa Cruz Biotechnology, Inc.); mouse monoclonal IgG1 to phosphoinositide 3-kinase p85 $\alpha$ (PI3K; 1:200; cat no. sc-1637; Santa Cruz Biotechnology,Inc.), rabbit polyclonal IgG to p-mammalian target of rapamycin (mTOR; 1:100; cat no. sc-8319; Santa Cruz Biotechnology, Inc.), rabbit polyclonal anti-Beclin-1 (1:5,000; cat no. ab55878; Abcam, Cambridge, MA, USA) and rabbit monoclonal to p-mitogen-activated protein kinase 8 (JNK1; 1:100; cat no. EPR17557; Abcam). Membranes were then washed four times for 5 min with Tris-buffered saline containing Tween 20, followed by incubation with the corresponding secondary antibodies, goat anti-rabbit immunoglobulin (Ig)G-HRP and goat anti-mouse IgG-HRP (1:5,000; cat. nos. A0208 and A0216, respectively; Beyotime Institute of Biotechnology), for $90 \mathrm{~min}$ at room temperature. For visualization, the membranes were treated with an enhanced chemiluminescence solution (ECL, Qihai Biotec, Shanghai, China), exposed to X-ray film and visualized by autoradiography. The optical density (OD) values of the target protein bands were analyzed using Gel-Pro-Analyzer software 4.0 (Media Cybernetics, Rockville, MD, USA) and normalized to $\beta$-actin (1:5,000; HRP-conjugated monoclonal mouse anti- $\beta$-actin; cat. no. KC-5A08; KangChen Bio-tech Inc., Shanghai, China).

Detection of cell viability using an MTT assay. Following transfection for $48 \mathrm{~h}$, cells were seeded onto 96-well plates at $1 \times 10^{3}$ cells/well and treated with various concentrations of memantine (1,5 and $10 \mu \mathrm{M})$; each experiment was performed with five replicates. After 24 and $48 \mathrm{~h}$, MTT solution (final concentration, $0.2 \mathrm{mg} / \mathrm{ml}$; Sigma-Aldrich) was added and incubated with the cells for $5 \mathrm{~h}$. The supernatant was carefully discarded and $200 \mu$ l DMSO (Sigma-Aldrich) was added to fully dissolve the crystals. OD values were measured at $490 \mathrm{~nm}$ using a microplate reader (ELX800; BioTek Instruments, Inc., Winooski, VT, USA).

Observation of autophagosomes using acridine orange. Following $24 \mathrm{~h}$ transfection, the cells were seeded in 12-well 
A

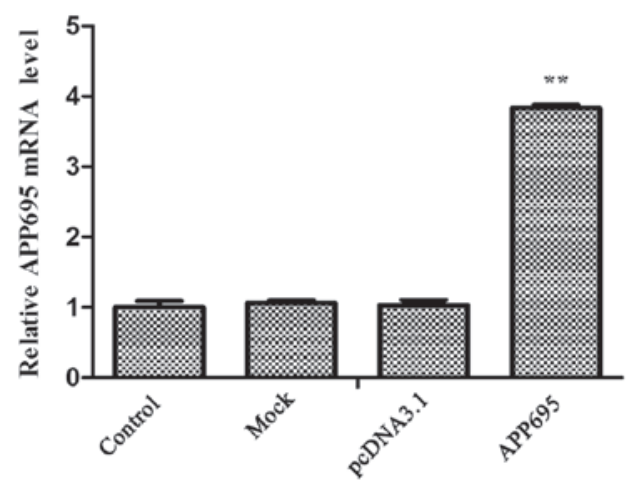

B

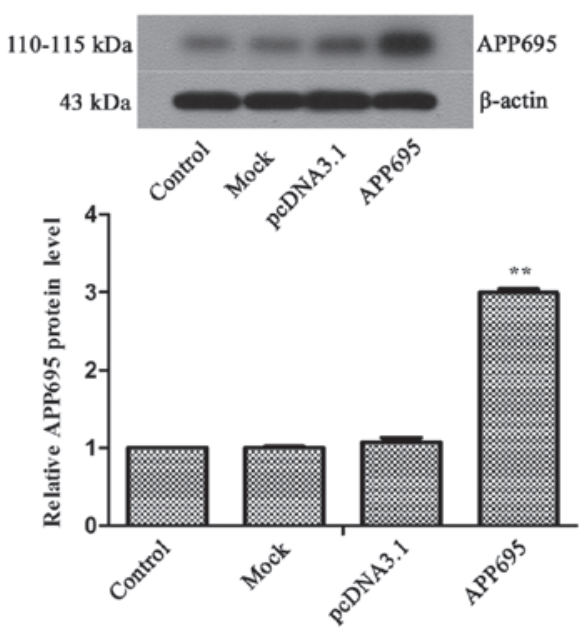

Figure 1. Establishment of an Alzheimer's disease cell model that overexpresses APP695. (A) Detection of APP695 mRNA expression levels using reverse transcription-quantitative polymerase chain reaction. (B) Detection of APP695 protein expression levels using western blot analysis. Typical results of repeated experiments are shown. All experiments were repeated in triplicate. $\beta$-actin was used as an internal control. Values are expressed as the mean \pm standard deviation. ${ }^{* *} \mathrm{P}<0.01$, vs. the pcDNA3.1 group. Groups: Control, untreated cells; pcDNA3.1, cells transfected with empty vector; Mock, cells treated with transfection reagent only; APP695, cells transfected with APP695 overexpression vector. APP695, $\beta$-amyloid precursor protein with 695-amino-acid mutation.

plates at a density of $1 \times 10^{5}$ cells/well. Following culturing in the presence of the corresponding drugs, the cells from each group were fixed in $4 \%$ paraformaldehyde (China National Medicines Corporation, Ltd.) for $15 \mathrm{~min}$ and washed four times with phosphate-buffered saline (PBS). Acridine orange (Beijing Solarbio Science \& Technology Co., Ltd., Beijing, China) solution $(0.01 \%)$ was added onto the cells in order to completely cover them; the cells were then incubated at room temperature for $5 \mathrm{~min}$. The stained cells were then mounted with an anti-fluorescein quencher (Beijing Solarbio Science \& Technology Co., Ltd.) and were observed under a laser scanning confocal microscope (FV1000S-SIM/IX81; Olympus Corp., Tokyo, Japan) and images were captured.

Detection of apoptosis using flow cytometry. Following $24 \mathrm{~h}$ of transfection, the cells were seeded into six-well plates at $1 \times 10^{5}$ cells/well. The cells from each group were collected following drug treatment and washed twice with PBS. The cells were then re-suspended in $500 \mu$ l binding buffer and mixed sequentially with $5 \mu \mathrm{l}$ Annexin V-allophycocyanin (APC) and $5 \mu 1$ 7-aminoactinomycin D (7-AAD) using an Annexin V-APC/7-AAD Apoptosis Detection kit (cat no. KGA1025; Nanjing KeyGen Biotech Co., Ltd., Nanjing, China), according to the manufacturer's instructions. The cells were subsequently incubated at room temperature in the dark for $15 \mathrm{~min}$, and apoptotic rates were detected using a FACSCalibur flow cytometer (BD Biosciences, Franklin Lakes, USA). The data were analyzed using CellQuest Pro 3.3 software (BD Biosciences).

Immunofluorescence double staining. Following $48 \mathrm{~h}$ of transfection, the cells were plated onto coverslips in six-well plates. Following cellular treatment with each group of drugs, the cells on the coverslips from each group were fixed in $4 \%$ paraformaldehyde for $15 \mathrm{~min}$ and permeabilized in $0.1 \%$ Triton X-100 (Amresco, Solon, OH, USA) for $30 \mathrm{~min}$. The cells were incubated with anti-Bcl-2 (1:200) and Beclin-1
(1:100) primary antibodies at $4^{\circ} \mathrm{C}$ overnight. The cells were then incubated with cyanine 3-labeled goat anti-mouse IgG $(\mathrm{H}+\mathrm{L})$ and fluorescein isothiocyanate-labeled goat anti-rabbit IgG (H+L) secondary antibodies (cat nos. A0521 and A0562, respectively; Beyotime Institute of Biotechnology) at a dilution of 1:100 for $90 \mathrm{~min}$ at room temperature. After staining with DAPI (Biosharp, Anhui, China), the coverslips were mounted inversely on slides with an anti-fluorescein quencher and were observed under a laser-scanning confocal microscope (Olympus Corporation) and the images were captured.

Statistical analysis. All values are expressed as the mean \pm standard deviation. The data were analyzed using Graphpad Prism 5.0 (GraphPad Software, Inc., La Jolla, CA, USA). Comparisons between two groups were performed using a one-way analysis of variance, whereas the comparisons among multiple groups were performed with Bonferroni's post hoc test. $\mathrm{P}<0.05$ was considered to indicate a statistically significant difference.

\section{Results}

Establishment and identification of an AD cell model overexpressing APP695 Swedish mutant gene. To establish an AD cell model, a pcDNA3.1-APP695 plasmid overexpressing the APP695 Swedish mutant gene was transfected into SH-SY5Y cells, and the expression levels of APP695 in the cells were detected using RT-qPCR and western blot analyses. The mRNA and protein expression levels of APP695 in the APP695 transfection group were 3.83-fold (Fig. 1A; $\mathrm{P}<0.01$ ) and 2.99-fold (Fig. 1B; $\mathrm{P}<0.01$ ) higher, respectively, as compared with those in the pcDNA3.1 group. These results suggested that the AD cell model was successfully established.

Memantine enhances neuronal cell viability and inhibits autophagy in a cell model of $A D$. The effects of various concentrations of memantine on neuronal cell viability in the 

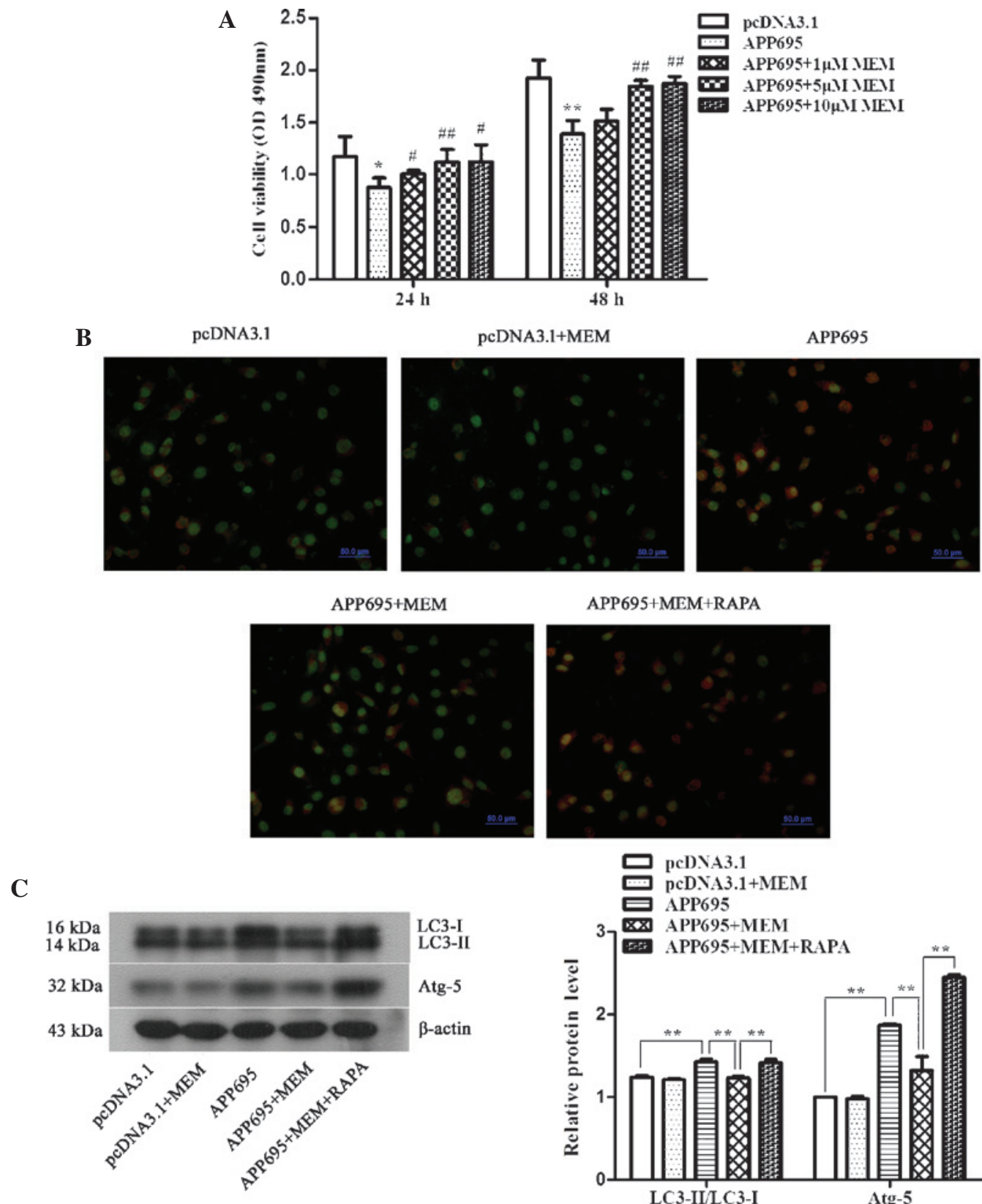

APP695+MEM+RAPA
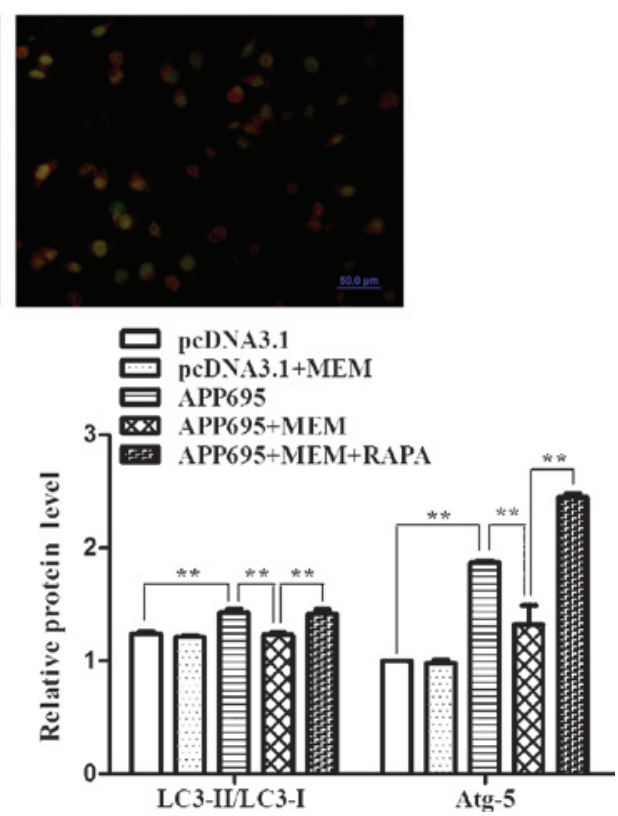

Figure 2. Increase in neuronal cell viability and inhibition of cell autophagy in the Alzheimer's disease cell model following treatment with memantine. (A) Detection of cell viability using an MTT assay. Cells were inoculated onto 96-well plates and treated with 1,5 or $10 \mu \mathrm{M}$ memantine. The absorbance values $\left(\mathrm{OD}, 490 \mathrm{~nm}\right.$ ) of the cells from each group were measured at 24 and $48 \mathrm{~h} .{ }^{*} \mathrm{P}<0.05$ and ${ }^{* * *} \mathrm{P}<0.01$, vs. the pcDNA3.1 group; ${ }^{*} \mathrm{P}<0.05$ and ${ }^{\# \prime} \mathrm{P}<0.01$, vs. the APP695 group. (B) Detection of autophagosomes using acridine orange staining. The cells were inoculated onto coverslips in 12-well plates. Following treatment with the indicated drugs for $24 \mathrm{~h}$, the cells were stained with $0.01 \%$ acridine orange. The results were observed under a laser scanning confocal microscope (magnification, $\mathrm{x} 400$; scale bar, $50 \mu \mathrm{m}$ ), and the most representative images are shown. Normal cells exhibit green fluorescence and autophagosomes exhibit orange-red fluorescence. (C) Detection of LC3-II/LC3-I and ATG5 protein expression levels as determined by western blot analysis. $\beta$-actin was used as an internal control. Representative results from three experiments repeated in triplicate are shown. ${ }^{* *} \mathrm{P}<0.01$. All of the above experiments were repeated in triplicate and all values are expressed as the mean \pm standard deviation. Groups: pcDNA3.1, cells transfected with empty vector; APP695, cells transfected with APP695 overexpression vector; memantine, cells treated with $5 \mu \mathrm{M}$ memantine for $24 \mathrm{~h}$; RAPA, cells were pre-treated with $0.2 \mu \mathrm{M}$ RAPA for $24 \mathrm{~h}$ prior to treatment with memantine. LC3, protein light chain 3; ATG-5, autophagy protein 5; OD, optical density; APP695, $\beta$-amyloid precursor protein with 695-amino-acid mutation; RAPA, rapamycin.

AD model were detected using an MTT assay. The results indicated that cell viability in the APP695 group at 24 (Fig. 2A; $\mathrm{P}<0.05)$ and $48 \mathrm{~h}(\mathrm{P}<0.01)$ were significantly decreased, as compared with those in the pcDNA3.1 group. However, memantine reversed the inhibition of cell proliferation caused by APP695 overexpression. Cell viability was significantly increased following cellular treatment with three different doses of memantine for $24(5 \mu \mathrm{M}, \mathrm{P}<0.01 ; 1 \mu \mathrm{M}$ and $10 \mu \mathrm{M}$, $\mathrm{P}<0.05)$ and $48 \mathrm{~h}(5 \mu \mathrm{M}$ and $10 \mu \mathrm{M}, \mathrm{P}<0.01)$, as compared with that in the APP695 group. However, no statistically significant difference was observed between the $5 \mu \mathrm{M}$-treated group and the $10 \mu \mathrm{M}$-treated group. Therefore, $5 \mu \mathrm{M}$ memantine was the optimal treatment concentration, and was used in the subsequent experiments.

To determine the effects of memantine on the autophagic capacity of neuronal cells in the AD model, the acridine orange staining method was used to observe autophagosomes, and western blotting was performed to detect the expression 
A
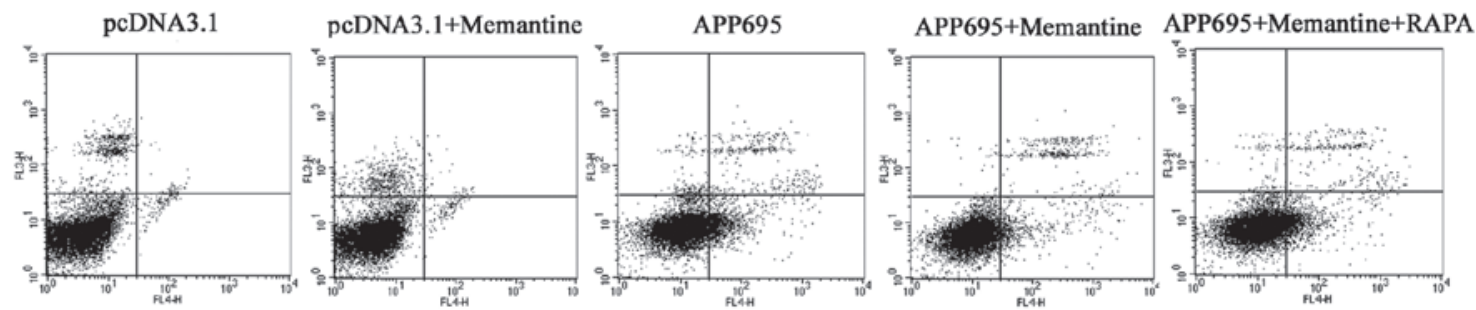

B

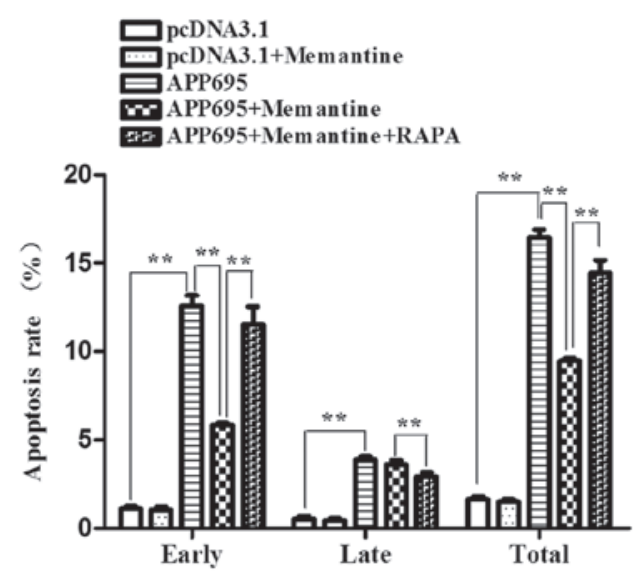

C

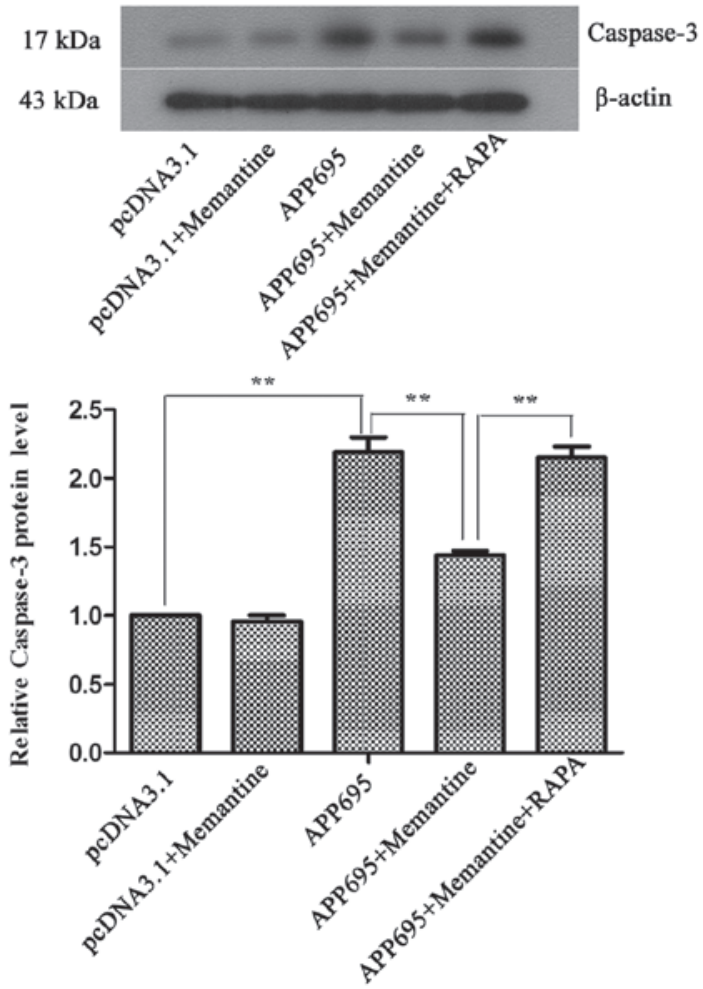

Figure 3. Inhibition of neuronal apoptosis in the Alzheimer's disease cell model following treatment with memantine, and promotion of cell apoptosis by rapamycin-induced autophagy (A) Detection of cell apoptosis using flow cytometry. The cells were seeded onto six-well plates and treated with the indicated drugs for $24 \mathrm{~h}$. Following double-staining with Annexin V-allophycocyanine/7-aminoactinomycin D, the cells were analyzed using flow cytometry. (B) Apoptotic rates in each group. (C) Detection of the protein expression levels of caspase-3 using western blotting. $\beta$-actin was used as an internal control. Representative results from three experiments repeated in triplicate are shown. ${ }^{* *} \mathrm{P}<0.01$. Groups: pcDNA3.1, cells transfected with empty vector; APP695, cells transfected with APP695 overexpression vector; memantine, cells treated with memantine for 24 h; RAPA, cells were pre-treated with $0.2 \mu$ M RAPA for $24 \mathrm{~h}$ prior to treatment with $5 \mu \mathrm{M}$ memantine. APP695, $\beta$-amyloid precursor protein with 695-amino-acid mutation; RAPA, rapamycin.

levels of the autophagy-associated proteins LC3-II/LC3-I and ATG5. Acridine orange is a weak base that has the ability to move freely across biological membranes in an uncharged state and is characterized by green fluorescence. Acridine orange, in its protonated form, accumulates in acidic compartments and forms aggregates, which are characterized by yellow-orange fluorescence. As shown in Fig. 2B, cells in the pcDNA3.1 group exhibited low levels of green fluorescence, indicating a lack of acidic vesicular organelles (AVOs). Conversely, APP695 overexpression induced the formation of yellow-orange fluorescent AVOs. The LC3-II/LC3-I ratio (Fig. 2C, P<0.01) and the expression levels of ATG5 $(\mathrm{P}<0.01)$ in the APP695 group were significantly increased. Following treatment with memantine, yellow-orange fluorescence, the LC3-II/LC3-I ratio $(\mathrm{P}<0.01)$ and ATG5 expression levels $(\mathrm{P}<0.01)$ were significantly decreased. The RAPA pre-treatment group showed a statistically significant increase in yellow-orange fluorescence, the LC-3II/LC3-I ratio $(\mathrm{P}<0.01)$ and the ATG5 expression levels $(\mathrm{P}<0.01)$.
Memantine inhibits neuronal apoptosis in a cell model of $A D$ and RAPA-induced autophagy promotes cell apoptosis. To detect the effects of memantine on neuronal apoptosis in the AD model and to clarify the association between autophagy and apoptosis, the apoptotic rates of the cells in each group were determined using flow cytometry following Annexin V-APC/7-AAD double staining. The expression levels of the apoptosis-associated protein caspase-3 were detected by western blot analysis. The apoptotic rates of the cells in the APP695 group were significantly increased, as compared with those in the pcDNA3.1 group (Fig. $3 \mathrm{~A}$ and $\mathrm{B} ; \mathrm{P}<0.01$ ); the expression levels of caspase-3 also increased by 2.19 -fold (Fig. 3C; $\mathrm{P}<0.01)$. Memantine treatment significantly decreased the apoptotic rate $(\mathrm{P}<0.01)$ and caspase-3 expression compared with those in the APP695 group $(\mathrm{P}<0.01)$, indicating that memantine was able to effectively inhibit neuronal apoptosis induced by APP695 overexpression. When the cells were pre-treated with RAPA, autophagy induced by RAPA significantly increased the apoptosis rates and promoted neuronal apoptosis $(\mathrm{P}<0.01)$. 
A

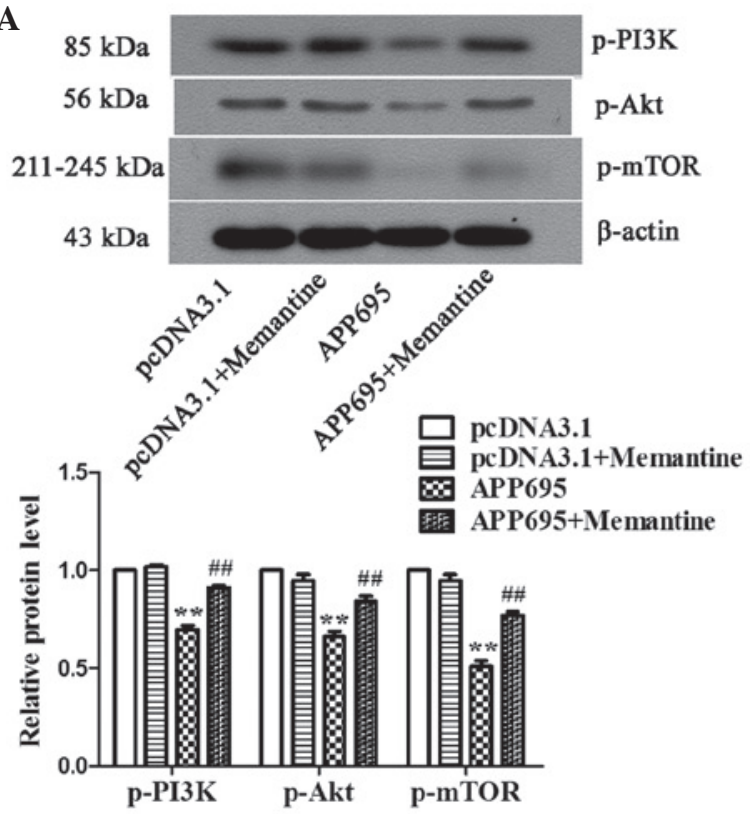

C

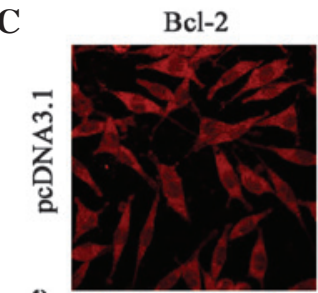

党
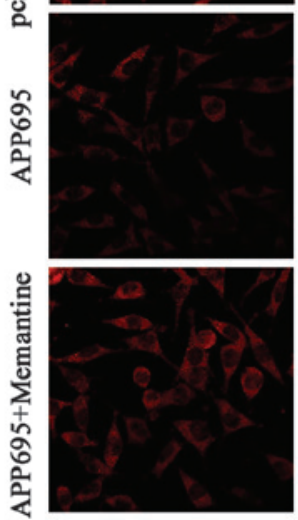

Beclin-1
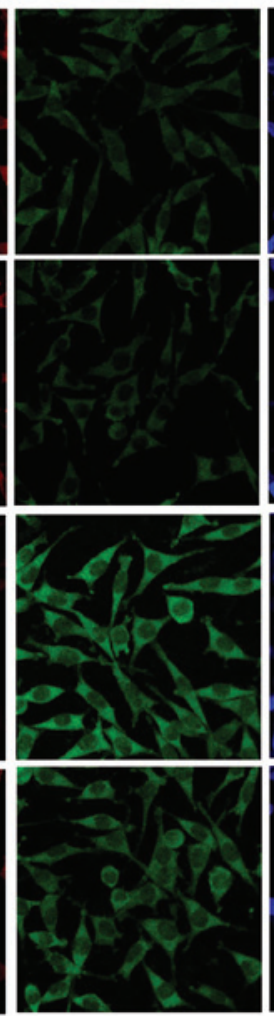

B
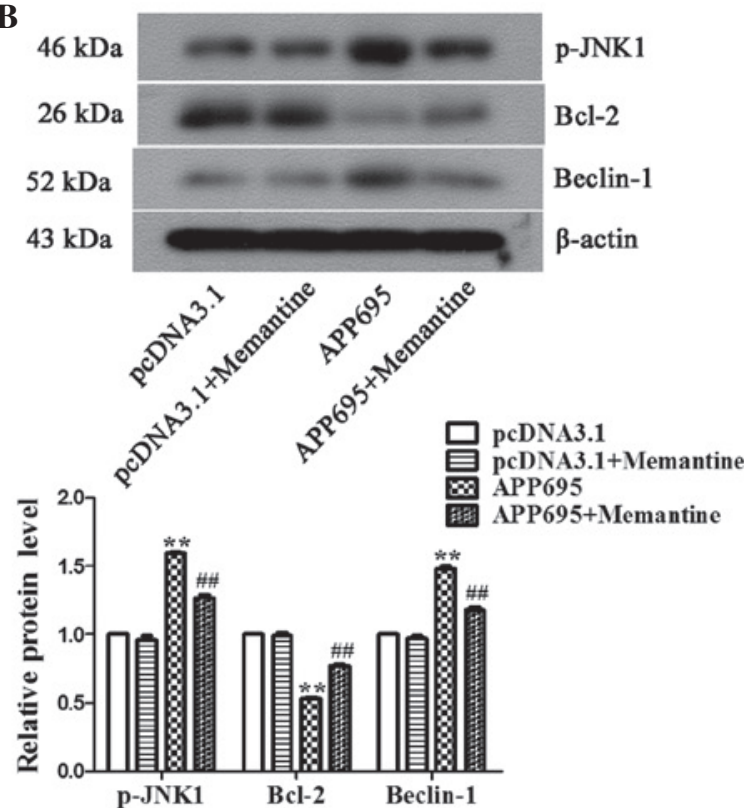

DAPI

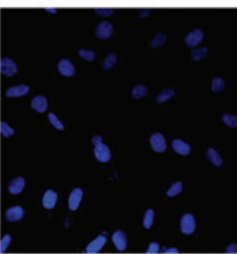

Merged

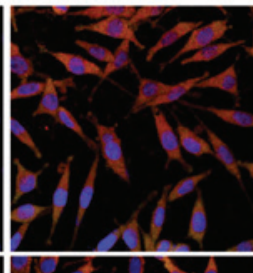

PP 695

APP695+Memantine
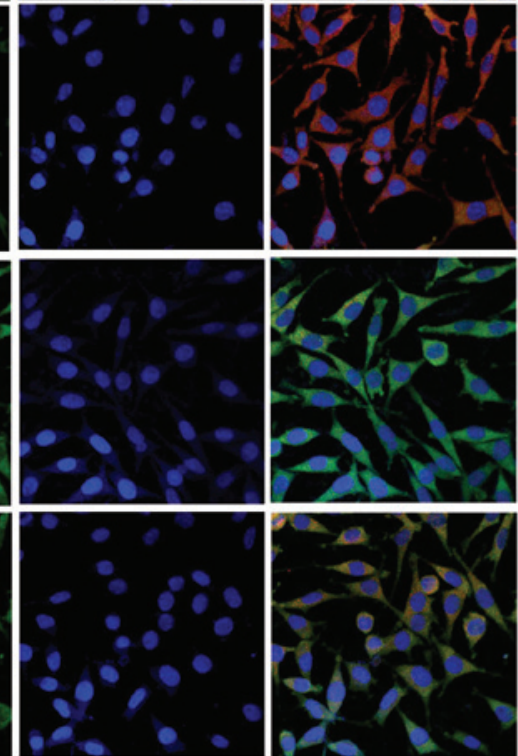

Figure 4. Regulation of autophagy-associated signaling pathways in neuronal cells by memantine. (A) Detection of the expression levels of PI3K, Akt and mTOR in the mTOR-dependent signaling pathway, as determined by western blot analysis. (B) Detection of the expression of p-JNK1, Bcl-2 and Beclin-1 in the mTOR-independent pathway, as determined by western blot analysis. (C) Observation of the expression of the Bcl-1/Beclin-1 complex using immunofluorescence. Bcl-2 stained with Cy3-labeled goat anti-mouse IgG (red), Beclin-1 stained with FITC-labeled goat anti-rabbit IgG (green) and cell nuclei stained with DAPI (blue) in the cells were observed under a laser scanning confocal microscope (magnification, x600). Representative data/images of three independent experiments are shown. Values are expressed as the mean \pm standard deviation. ${ }^{* *} \mathrm{P}<0.01$, vs. the pcDNA3.1 group; ${ }^{\# \#} \mathrm{P}<0.01$ vs. the APP695 group. Groups: pcDNA3.1, cells transfected with empty vector; APP695, cells transfected with APP695 overexpression vector; memantine, cells treated with $5 \mu$ M memantine for 24 h. P, phosphorylated; PI3K, phosphoinositide 3-kinase; mTOR, mammalian target of rapamycin; JNK1, mitogen-activated protein kinase 8; Bcl-2, B cell lymphoma 2; Cy3, cyanine 3; IgG, immunoglobulin G, FITC, fluorescein isothiocyanate.

Memantine regulates autophagic signaling pathways in neuronal cells. The mechanisms underlying the inhibition of autophagy by memantine were determined by detecting the activation of the two mTOR-dependent and -independent signaling pathways. Western blot analysis demonstrated that the expression levels of p-PI3K, p-Akt and p-mTOR in the APP695 group were significantly downregulated, as compared with those in the pcDNA3.1 group (Fig. 4A; $\mathrm{P}<0.01$ ). Following treatment of 
the cells in the APP695 group with memantine, the expression levels of the three signaling molecules significantly increased $(\mathrm{P}<0.01)$, suggesting that APP695 overexpression inhibited the $\mathrm{PI} 3 \mathrm{~K} / \mathrm{Akt} / \mathrm{mTOR}$ signaling pathway in neuronal cells and to activate autophagy, whereas memantine reversed the inhibitory state of the PI3K/Akt/mTOR signaling pathway, suppressing autophagy. In the mTOR-independent signaling pathway, p-JNK1 and Beclin-1 expression levels were significantly upregulated in the APP695 group, as compared with those in the pcDNA3.1 group (Fig. 4B; $\mathrm{P}<0.01$ ), whereas the expression levels of $\mathrm{Bcl}-2$ expression were significantly downregulated $(\mathrm{P}<0.01)$, which was attenuated by treatment with memantine. Immunofluorescence staining was performed to further investigate the expression of the Bcl-2/Beclin-1 complex. The results indicated that the localizations of the Bcl-2 and Beclin-1 proteins in the cells were matched. The Bcl-2/Beclin-1 complex in the pcDNA3.1 group and the pcDNA3.1 + Memantine group exhibited orange-yellow fluorescence in the cytoplasm. In the APP695 group, Bcl-2 was phosphorylated, and its expression levels were decreased; therefore, the cytoplasm emitted emerald green fluorescence. In the APP695 + Memantine group, the expression of the Bcl-2/Beclin-1 complex was increased and the color of the fluorescence in the cytoplasm had returned to orange-yellow, similar to that in the empty vector-transfected cells. These results indicated that memantine activated the Bcl-2/Beclin-1 complex to inhibit autophagy in the AD cell model via the suppression of JNK1 phosphorylation. In conclusion, the above results demonstrated that the mTOR-dependent as well as -independent pathways are involved in the inhibition of cell autophagy by memantine.

\section{Discussion}

Blocking of NMDA receptors by memantine is an effective method for the treatment of $\mathrm{AD}$; however, its effects on neuronal autophagy and apoptosis in AD have remained to be elucidated. The present study established an AD cell model that overexpressed APP695. The results indicated that memantine increased cell viability in the AD model. Memantine exerted its anti-autophagic and anti-apoptotic functions via the mTOR-dependent and mTOR-independent signaling pathways. These results preliminarily explored the mechanisms underlying the anti-autophagic effects of memantine. In addition, pre-treatment of the AD cell model with the autophagy enhancer RAPA demonstrated that autophagy promoted cell apoptosis. These results provided experimental evidence regarding the controversial issue of the association between autophagy and apoptosis.

An increase in autophagosome formation (28) and activation of caspases was detected (29) in the neurons of AD patients. Numerous studies have reported that $A \beta$ accumulation induced NMDA receptor overactivation $(30,31)$, whereas NMDA activation promoted neuronal autophagy or apoptosis. Gao et al (32) treated rat cardiomyocytes with the NMDA receptor antagonist MK-801, thereby demonstrating that apoptosis in cardiomyocytes was significantly improved following treatment, and that the expression levels of the apoptosis-associated proteins $\mathrm{Bcl}-2$-associated $\mathrm{X}$ protein and caspase-3 decreased significantly. Therefore, these results led to the speculation that memantine, which is an NMDA receptor antagonist, may inhibit neuronal autophagy and apoptosis. The present study established an AD cell model by upregulating APP695 expression and demonstrated that memantine increased the viability of SH-SY5Y cells overexpressing APP695. In addition, $5 \mu \mathrm{M}$ memantine significantly inhibited cell autophagy and apoptosis. However, these inhibitory effects were reversed by pre-incubation with RAPA.

In $\mathrm{AD}$ research, the association between neuronal autophagy and apoptosis is controversial. Nilsson et al (19) reported that APP and its hydrolytic enzymes were not degraded by autophagosomes following the conversion of autophagosomes, thereby leading to the production of large amounts of $A \beta(17,19,20)$ and an increase in cell apoptosis. Spilman et al (21) reported that enhancement of autophagy reduced the levels of $A \beta$, thereby inhibiting apoptosis (21-24). In the present study, AD model cells were pre-treated with the autophagy enhancer RAPA and were compared with cells in other treatment groups. The results demonstrated that the enhancement of neuronal autophagy significantly increased the apoptotic rates of the cells, which was concordant with the results of Nilsson et al (19).

mTOR is an important regulator of autophagy, and the mTOR-dependent signaling pathway is regulated by $\mathrm{PI} 3 \mathrm{~K} / \mathrm{Akt} / \mathrm{mTOR}$. Inhibition of this signaling pathway may activate cell autophagy (33-36). In the mTOR-independent signaling pathway, Bcl-2 is phosphorylated via the protein kinase JNK1 to separate Bcl-2 and Beclin-1; autophagy is then activated by Beclin-1 (37). The present study demonstrated that memantine was able to significantly upregulate the expression of the signaling factors PI3K, Akt and mTOR, to activate the mTOR-dependent autophagy signaling pathway, and to reduce the autophagic capacity of neuronal cells. In addition, the present study also detected that memantine inhibited the phosphorylation of the Bcl-2/Beclin-1 complex by JNK1 to prevent the activation of cell autophagy by Beclin-1. These results suggested that the two signaling pathways were involved in the inhibition of cell autophagy by memantine.

In conclusion, the results of the present study demonstrated that memantine exerted significant anti-autophagic and anti-apoptotic effects in an APP695-overexpressing cell model of AD, the mechanism of which involved the mTOR-dependent as well as -independent autophagic signaling pathways. The present study supported the clinical use of memantine for the treatment of AD. Furthermore, the present study confirmed that autophagy in the AD cell model was able to promote cell apoptosis, thus providing experimental evidence regarding the association between autophagy and apoptosis.

\section{References}

1. Ballard C, Gauthier S, Corbett A, Brayne C, Aarsland D and Jones E: Alzheimer's disease. Lancet 377: 1019-1031, 2011.

2. Huang Y and Mucke L: Alzheimer mechanisms and therapeutic strategies. Cell 148: 1204-1222, 2012.

3. De Felice FG, Velasco PT, Lambert MP, Viola K, Fernandez SJ, Ferreira ST and Klein WL: Abeta oligomers induce neuronal oxidative stress through an N-methyl-D-aspartate receptor-dependent mechanism that is blocked by the Alzheimer drug memantine. J Biol Chem 282: 11590-11601, 2007.

4. Texidó L, Martín-Satué M, Alberdi E, Solsona C and Matute C: Amyloid $\beta$ peptide oligomers directly activate NMDA receptors. Cell Calcium 49: 184-190, 2011. 
5. Zheng L, Kågedal K, Dehvari N, Benedikz E, Cowburn R, Marcusson J and Terman A: Oxidative stress induces macroautophagy of amyloid beta-protein and ensuing apoptosis. Free Radic Biol Med 46: 422-429, 2009.

6. Lorenzi M, Beltramello A, Mercuri NB, Mercuri NB, Canu E, Zoccatelli G, Pizzini FB, Alessandrini F, Cotelli M, Rosini S, et al: Effect of memantine on resting state default mode network activity in Alzheimer's disease. Drugs Aging 28: 205-217, 2011.

7. Saxton J, Hofbauer RK, Woodward M, Gilchrist NL, Potocnik F, Hsu HA, Miller ML, Pejović V, Graham SM and Perhach JL: Memantine and functional communication in Alzheimer's disease Results of a 12-week, international, randomized clinical trial. J Alzheimers Dis 28: 109-118, 2012.

8. Schulz JB, Rainer M, Klünemann HH, Kurz A, Wolf S, Sternberg K and Tennigkeit F: Sustained effects of once-daily memantine treatment on cognition and functional communication skills in patients with moderate to severe Alzheimer's disease: Results of a 16-week open-label trial. J Alzheimers Dis 25: 463-475, 2011.

9. Liu MY, Wang S, Yao WF, Zhang ZJ, Zhong X, Sha L, He M Zheng ZH and Wei MJ: Memantine improves spatial learning and memory impairments by regulating NGF signaling in APP/PS1 transgenic mice. Neuroscience 273: 141-151, 2014.

10. Danysz W and Parsons CG: The NMDA receptor antagonist memantine as a symptomatological and neuroprotective treatment for Alzheimer's disease: Preclinical evidence. Int J Geriatr Psychiatry (Suppl 1) 18: S23-S32, 2003.

11. Peskind ER, Potkin SG, Pomara N, Ott BR, Graham SM, Olin JT and McDonald S: Memantine treatment in mild to moderate Alzheimer disease: A 24-week randomized, controlled trial. Am J Geriatr Psychiatry 14: 704-715, 2006.

12. Ray B, Banerjee PK, Greig NH and Lahiri DK: Memantine treatment decreases levels of secreted Alzheimer's amyloid precursor protein (APP) and amyloid beta (A beta) peptide in the human neuroblastoma cells. Neurosci Lett 470: 1-5, 2010

13. Alley GM, Bailey JA, Chen D, Ray B, Puli LK, Tanila H, Banerjee PK and Lahiri DK: Memantine lowers amyloid-beta peptide levels in neuronal cultures and in APP/PS1 transgenic mice. J Neurosci Res 88: 143-154, 2010.

14. Maekawa M, Namba T, Suzuki E, Yuasa S, Kohsaka S and Uchino S: NMDA receptor antagonist memantine promotes cell proliferation and production of mature granule neurons in the adult hippocampus. Neurosci Res 63: 259-266, 2009.

15. Namba T, Maekawa M, Yuasa S, Kohsaka S and Uchino S: The Alzheimer's disease drug memantine increases the number of radial glia-like progenitor cells in adult hippocampus. Glia 57 1082-1090, 2009.

16. NambaT, Yabe T, Gonda Y, et al: Pigment epithelium-derived factor up-regulation induced by memantine, an $\mathrm{N}$-methyl-D-aspartate receptor antagonist, is involved in increased proliferation of hippocampal progenitor cells. Neuroscience 167: 372-383, 2010.

17. Ghavami S, Shojaei S, Yeganeh B, Ande SR, Jangamreddy JR, Mehrpour M, Christoffersson J, Chaabane W, Moghadam AR, Kashani HH, et al: Autophagy and apoptosis dysfunction in neurodegenerative disorders. Prog Neurobiol 112: 24-49, 2014

18. Nixon RA: Autophagy, amyloidogenesis and Alzheimer disease. J Cell Sci 120: 4081-4091, 2007.

19. Nilsson P, Loganathan K, Sekiguchi M, Matsuba Y, Hui K, Tsubuki S, Tanaka M, Iwata N, Saito T and Saido TC: A $\beta$ secretion and plaque formation depend on autophagy. Cell Reports 5: 61-69, 2013.

20. Yu WH, Cuervo AM, Kumar A, Peterhoff CM, Schmidt SD, Lee JH, Mohan PS, Mercken M, Farmery MR, Tjernberg LO, et al: Macroautophagy--a novel Beta-amyloid peptide-generating pathway activated in Alzheimer's disease. J Cell Biol 171: 87-98, 2005.
21. Spilman P, Podlutskaya N, Hart MJ, Debnath J, Gorostiza O, Bredesen D, Richardson A, Strong R and Galvan V: Inhibition of mTOR by rapamycin abolishes cognitive deficits and reduces amyloid-beta levels in a mouse model of Alzheimer's disease. PLoS One 5: e9979, 2010.

22. Caccamo A, Majumder S, Richardson A, Strong R and Oddo S: Molecular interplay between mammalian target of rapamycin (mTOR), amyloid-beta, and Tau: Effects on cognitive impairments. J Biol Chem 285: 13107-13120, 2010.

23. Tan CC, Yu JT, Tan MS, Jiang T, Zhu XC and Tan L: Autophagy in aging and neurodegenerative diseases: Implications for pathogenesis and therapy. Neurobiol Aging 35: 941-957, 2014.

24. Zhu XC, Yu JT, Jiang T and Tan L: Autophagy modulation for Alzheimer's disease therapy. Mol Neurobiol 48: 702-714, 2013.

25. Ye X, Tai W, Bao X, Chen X and Zhang D: FLZ inhibited $\gamma$-secretase selectively and decreased $A \beta$ mitochondrial production in APP-SH-SY5Y cells. Naunyn Schmiedebergs Arch Pharmacol 387: 75-85, 2014

26. Shen YE, Wang Y, Yu GC, Liu C, Zhang ZY and Zhang LM: Effects of edaravone on amyloid- $\beta$ precursor protein processing in SY5Y-APP695 cells. Neurotox Res 24: 139-147, 2013.

27. Wang L, Zhang D, Yu Y, Guan H, Qiao C and Shang T: RNA interference-mediated silencing of laminin receptor 1 (LR1) suppresses migration and invasion and down-regulates matrix metalloproteinase (MMP)-2 and MMP-9 in trophoblast cells: Implication in the pathogenesis of preeclampsia. J Mol Histol 44: 661-668, 2013.

28. Nixon RA, Wegiel J, Kumar A, Yu WH, Peterhoff C, Cataldo A and Cuervo AM: Extensive involvement of autophagy in Alzheimer disease: An immuno-electron microscopy study. J Neuropathol Exp Neurol 64: 113-122, 2005.

29. Louneva N, Cohen JW, Han LY, albot K, Wilson RS, Bennett DA, Trojanowski JQ and Arnold SE: Caspase-3 is enriched in postsynaptic densities and increased in Alzheimer's disease. Am J Pathol 173: 1488-1495, 2008.

30. Kaufman AM, Milnerwood AJ, Sepers MD, Coquinco A, She K, Wang L, Lee H, Craig AM, Cynader M and Raymond LA: Opposing roles of synaptic and extrasynaptic NMDA receptor signaling in cocultured striatal and cortical neurons. J Neurosci 32: 3992-4003, 2012

31. Caballero B and Coto-Montes A: An insight into the role of autophagy in cell responses in the aging and neurodegenerative brain. Histol Histopathol 27: 263-275, 2012.

32. Gao X, Xu X, Pang J, Zhang C, Ding JM, Peng X, Liu Y and Cao JM: NMDA receptor activation induces mitochondrial dysfunction, oxidative stress and apoptosis in cultured neonatal rat cardiomyocytes. Physiol Res 56: 559-569, 2007.

33. Han W, Pan H, Chen Y, Sun J, Wang Y, Li J, Ge W, Feng L, Lin X, Wang X, et al: EGFR tyrosine kinase inhibitors activate autophagy as a cytoprotective response in human lung cancer cells. PLoS One 6: e18691, 2011.

34. Liu J,Hu XJ, Jin B, Qu XJ, Hou KZ and Liu YP: $\beta$-Elemene induces apoptosis as well as protective autophagy in human non-small-cell lung cancer A549 cells. J Pharm Pharmacol 64: 146-153, 2012.

35. Wang P, Guo QS, Wang ZW and Qian HX: HBX induces HepG-2 cells autophagy through PI3K/Akt-mTOR pathway. Mol Cell Biochem 372: 161-168, 2013.

36. Yang YH, Chen K, Li B, Chen JW, Zheng XF, Wang YR, Jiang SD and Jiang LS: Estradiol inhibits osteoblast apoptosis via promotion of autophagy through the ER-ERK-mTOR pathway. Apoptosis 18: 1363-1375, 2013.

37. Russell RC, Tian Y, Yuan H, Park HW, Chang YY, Kim J, Kim H, Neufeld TP, Dillin A and Guan KL: ULK1 induces autophagy by phosphorylating Beclin-1 and activating VPS34 lipid kinase. Nat Cell Biol 15: 741-750, 2013. 